\title{
Crown-Root Angulation of Central Incisors in Cases With Maxillary Lateral Incisors Agenesis
}

\author{
Alexandru Ogodescu (Assoc. Professor, PhD), \\ Ramona Ştefănescu (MD, Dentist), \\ Emilia Ogodescu (Assis. Professor, PhD), \\ Andreea Igna (Postgraduate Student), \\ Magda Luca (Assis. Professor, PhD)
}

Department of Paediatric Dentistry, Faculty of Dental Medicine, University of Medicine and Pharmacy "Victor Babeş", Timişoara, Romania

Sorin Bolintineanu (Assoc. Professor, PhD), Department of Anatomy and Embryology, University of Medicine and Pharmacy "Victor Babeş", Timişoara, Romania

doi: 10.19044/esj.2017.v13n15p330 URL:http://dx.doi.org/10.19044/esj.2017.v13n15p330

\begin{abstract}
Dental agenesis of the maxillary lateral incisor is a frequently seen anomaly in children and teenagers nowadays. It can alter the esthetics as well as the functions in the frontal area of the maxilla and therefore, early detection and therapy would be beneficial. The treatment often implies an interdisciplinary approach between orthodontics and implantology. One of the particularities of this kind of treatment is given by the distal angulation of central incisor's root, which invades the space required by the dental implant. Any treatment plan should begin with a thorough radiographical analysis of the space and morphological particularities of the central incisors.
\end{abstract}

Keywords: Lateral incisor agenesis, central incisor, distal root angulation

\section{Introduction}

Dental agenesis is a congenital anomaly, characterized by absence of dental units, which can affect both the primary and the permanent dentition. Normally, this anomaly regards the last tooth in each dental class, with the highest prevalence for the third molar, followed by the upper lateral incisor and the lower second premolar. The congenital absence of the maxillary lateral incisor, frequently seen in children and teenagers nowadays, alters the esthetics significantly. Therefore, treatment often requires an 
interdisciplinary approach between orthodontics and implantology. The diagnosis of certainty for this anomaly is established via radiological examination (Ștefănescu, Ogodescu, 2013).

Treating patients with maxillary lateral incisor agenesis implies choosing between two therapeutic options: opening and redistributing the space for a prosthetic restoration of the missing tooth (including the option of placing a dental implant), or closing the space by mesialising and morphologically reshaping the canine, as to resemble a lateral incisor.

The therapeutic option more frequently used in treating these patients consists of opening and resizing the space for implant placement. Consequently, it is essential to obtain an adequate space, both in the coronal area and in the apical region - which is often problematic. One of the problems that may occur in the apical region is the crown-root angulation of the central incisor, which diminishes the space required by the implant apically.

The aim of this study is to investigate whether patients with lateral incisor agenesis also present an altered crown-root morphology of the adjacent central incisors and to highlight the clinical and therapeutic changes that occur in such cases.

Tooth agenesis (hypodontia) is the most common craniofacial malformation, which can occur in association with other developmental anomalies (syndromic), or as an isolated condition (non-syndromic). Nonsyndromic agenesis can occur randomly, or can run in the family, by dominant/recessive autosomal transmission, or X-linked transmission. Syndromic congenital agenesis can be associated with over 49 disorders, out of which, more commonly seen are: cleft lip and palate, ectodermal dysplasia and Down syndrome. Association with other dental anomalies (microdontia, impaction of permanent canines, maxillary canine/first premolar transposition, taurodontism or even supernumerary teeth) was also recorded. Ethiopatogeny includes environmental factors, such as iradiation, chemotherapy, Thalidomid intake during pregnancy, malnutrition or neurological diseases, and genetic factors. Recent advances in genetic techniques have allowed so far the identification of two genes responsible for non-syndromic hypodontia: PAX9 and MSX1 (Pemberton et al., 2005; Larmour et al., 2005).

As a rule, agenesis in primary upper lateral incisors results in agenesis of the permanent successor. Moreover, when a mandibular primary lateral incisor and a canine are fused, agenesis of the permanent mandibular lateral incisor is a constant fact. In primary dentition, the incidence of agenesis shows no variation with sex, while in permanent dentition, a slight predisposition in female patients was observed. Also the maxilla is more 
affected than the madible. Racial differences have been determined and indicate a higher prevalence rate for lateral incisor agenesis within the Caucasian population (Stamatiou \& Symons, 1991; Larmour et al., 2005; Pinho, 2011).

Agenesis of any tooth can cause dental asymmetries, alignment difficulties, and arch length discrepancies but when the missing tooth is in the anterior region of the maxilla, the discrepancies can be quite noticeable (Azzaldeen et al., 2016). Patients and parents should be counseled about the complexities of this genetically inherited anomaly and the long-term treatment options (Abu-Hussein et al., 2014).

At the beginning of the treatment plan, a number of factors must be taken into consideration when deciding over space opening or closing procedures, such as the patient's age, profile, occlusion, esthetics and costs. While space-closing and canine substitution can be an excellent option for some Angle Class II patients (especially if they present an excessive overjet) (Kokich 2005), class III malocclusion is regarded as an inarguable indication for space opening and prosthetic restorations for the missing lateral incisors, as this can camouflage the existing malocclusion (Kinzer, 2005). As an observation, numerous patients with maxillary lateral incisor agenesis show a significant tendency for skeletal Class III. This could be attributed to maxillary hypoplasia/retrognathia (Bassiouny et al., 2016, Celikoglu et al., 2012). According to Kokich, there are three options when replacing a missing lateral incisor with a tooth-supported restoration: resin-bonded fixed partial denture - as a provisional option, a cantilevered fixed partial denture, or a conventional full-coverage fixed partial denture (Kokich \& Kinzer, 2005). The major disadvantage of this treatment option is that it commits the patient to a lifelong prosthesis in the most visible area of the mouth where tooth shade and transparency, gingival color, contour and margin levels are critical and difficult to control, particularly in the long term. Furthermore, the overall treatment is not complete when the orthodontic treatment ends, particularly in adolescent patients, who need long-term retention of the spaces with temporary retainers until skeletal growth and tooth eruption are complete. However, space opening for missing maxillary incisors has a set of indisputable advantages, functionally and for the occlusion, as it supports an ideal intercuspidation of canines through first premolars. A treatment plan that favors these teeth to be maintained in their natural position within the dental arch, with their natural morphology, is the placement of an osseointegrated implant (Abu-Hussein et al., 2014).

Replacing the missing lateral incisor with an implant is a prosthetic alternative which requires at least $6 \mathrm{~mm}$ of space (the smallest implant -of about $3 \mathrm{~mm}$ in diameter with at least $1 \mathrm{~mm}$ of bone between the implant and the added $1 \mathrm{~mm}$ crown restoration). In cases when clinicians cannot maintain 
the proper occlusion and create the minimum space for an implant, interproximal reduction (stripping) of the central incisors, canines, or premolars can be a solution (Azzaldeen et al., 2016; Kokich, 2004). A frequent problem which arises during the orthodontic treatment that aims to create space for the dental implant, is the distal root angulation of the maxillary central incisors, invading the apical space required by the implant. The upper central incisors occupy the main position in the esthetic zone, with a decisive role in physiognomy and phonation. Their normal mesio-distal inclination level is $5^{\circ}$. Unfortunately, in these teeth, a correct positioning of the coronal long axis does not always coincide with an adequate radicular axis. A thorough radiological examination of the roots of the central incisors at the beginning of the interdisciplinary treatment is therefore mandatory. Cone Beam Computed Tomography (CBCT) is indicated in such cases, as the $3 \mathrm{D}$ reconstruction of the anatomical structures offers more details of the angulation level, enhancing the accuracy of the measurements.

Kanavakis $\mathrm{G}$ et al. (2015) concluded that the root of lateral incisors adjacent to palatally impacted canines is angulated more mesially compared to lateral incisors adjacent to normally erupted canines. In addition, clinicians are somewhat able to predict if a canine is palatally impacted by visually observing the crown-to-root angulation of the adjacent lateral incisor. Evaluating the crown-to-root angulation of a lateral incisor on a panoramic image might facilitate an early diagnosis of palatally impacted canines.

In our retrospective, observational study, we investigate the differences in the morphology of central incisors, their crown-to-root angulation, in cases with lateral incisors agenesis. Clinical and radiological data were correlated in order to determine whether the space needs for an implant are met, both coronally and apically.

In order to verify this hypothesis, we examined 17 ortopantomographs of patients with bilateral incisors agenesis. In all these cases, the roots of the central incisors were completely formed and the apex closed as seen on X-ray. The following plans and axes were used as landmarks: reference plan represented by the incisal plane, long axis of the tooth crown so as to meet the degree of angulation stated by Andrews, the tooth root axis.

Following the tracing of these parts the angle between the crown and the root axis (the crown-root angulation) is determined (Fig.1). 


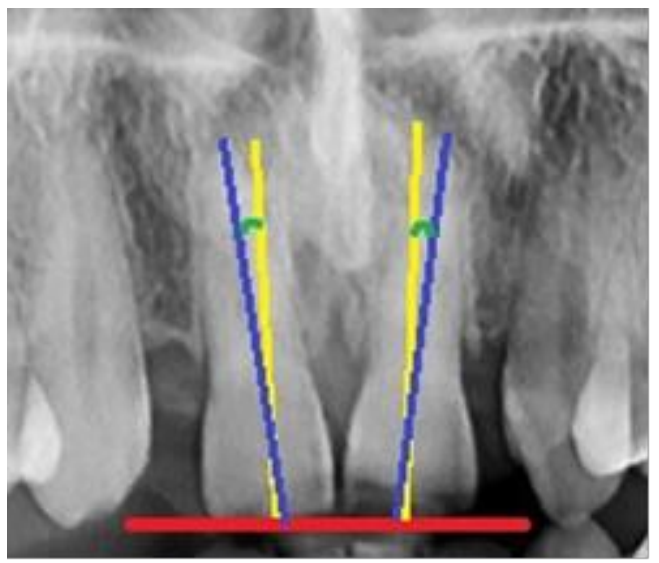

Fig. 1 Determination of the crown-root angulation: we draw the reference plane (red line), the crown axis (yellow line) and the root axis (blue line)

Some of the most representative cases with radiographic highlight to determine the crown-root angulation will be presented.

The radiographical examination of a 9-year old patient, with mixed dentition (Fig. 2), shows persistence of the upper lateral incisors on the arch, with roots unaffected by resorption, due to bilateral agenesis of permanent lateral incisors. Radicular angulation of the permanent central incisors is observed. Following measurements, a $7^{\circ}$ angulation of the root was determined in both teeth.

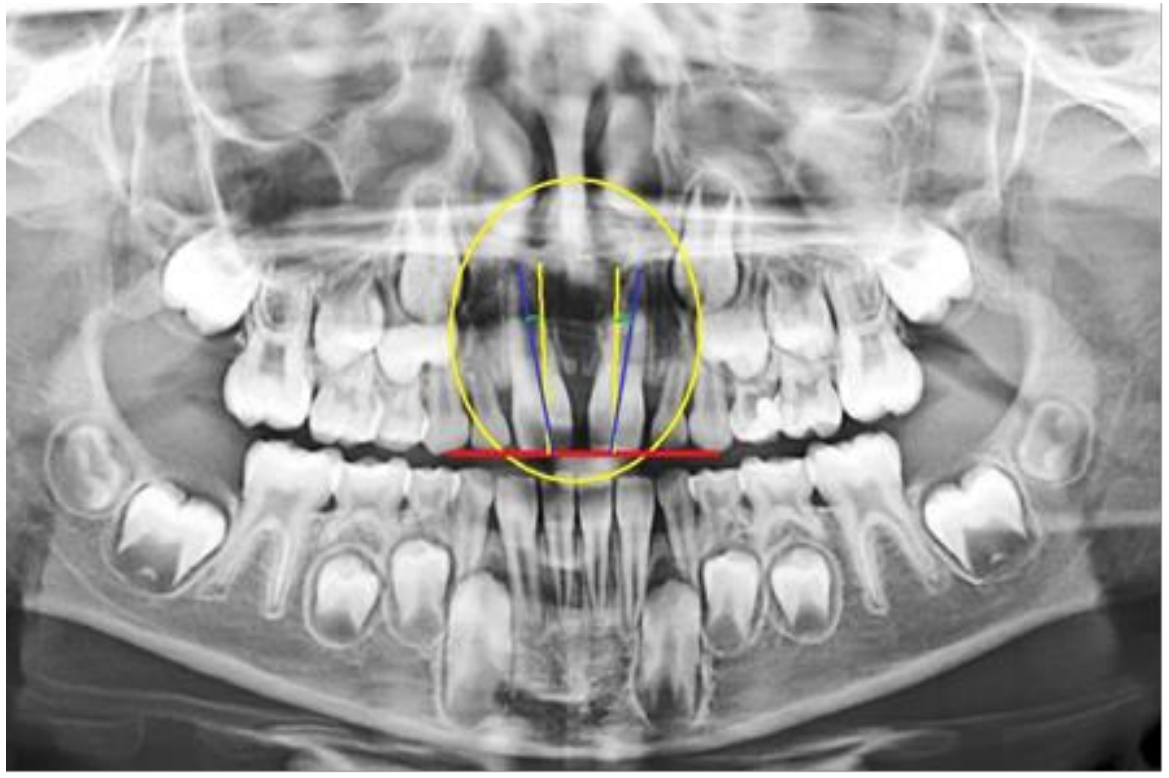

Fig. 2 X-ray of a 9-year old patient with agenesis of upper permanent lateral incisors and distal angulation of both central incisors'roots (The collection of the Paediatric Dentistry Discipline). 
The ortopantomography of a 28 -year old orthodontic patient, with permanent dentition (Fig. 3), displays an adequate angulation of the right upper central incisor and a considerable inclination of the left one. After measurements, a $14^{\circ}$ root angulation was proved, with consecutive reduction of space between the central incisor and the canine, resulting in a lack of space for implant placement in the upper left quadrant.

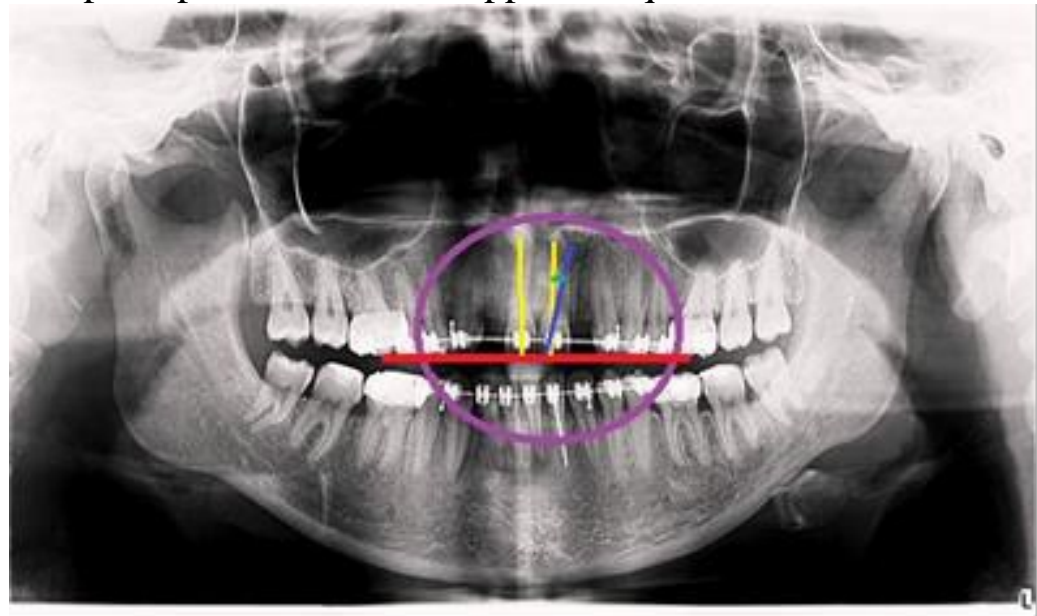

Fig. 3 X-ray of a 28-year old orthodontic patient with agenesis of upper lateral incisors, with a significant distal root angulation of the tooth 2.1

The CBCT 3D reconstruction of a patient with bilateral agenesis of permanent maxillary lateral incisors provides useful data regarding space, when implant therapy is the choice of treatment. Precise measurements can be carried out at both coronal and apical level, in order to determine the angulation degree of the teeth adjacent to the edentulous space, an aspect which dictates the suitability for implant placement (Fig. 4A,4B,4C).

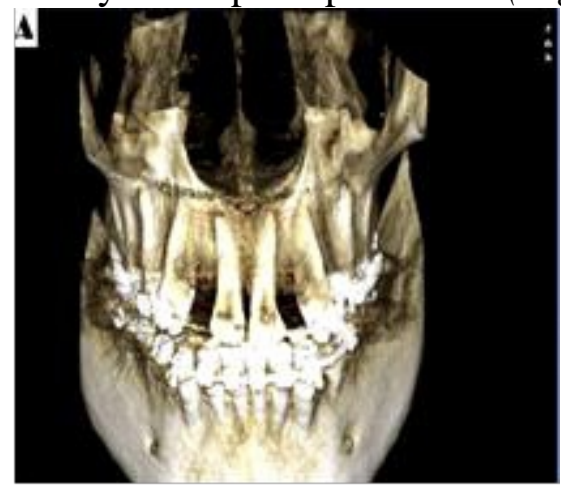

Fig. 4A) Tridimentional CBCT image of a patient with bilateral agenesis of permanent upper lateral incisors undergoing orthodontic treatment. 


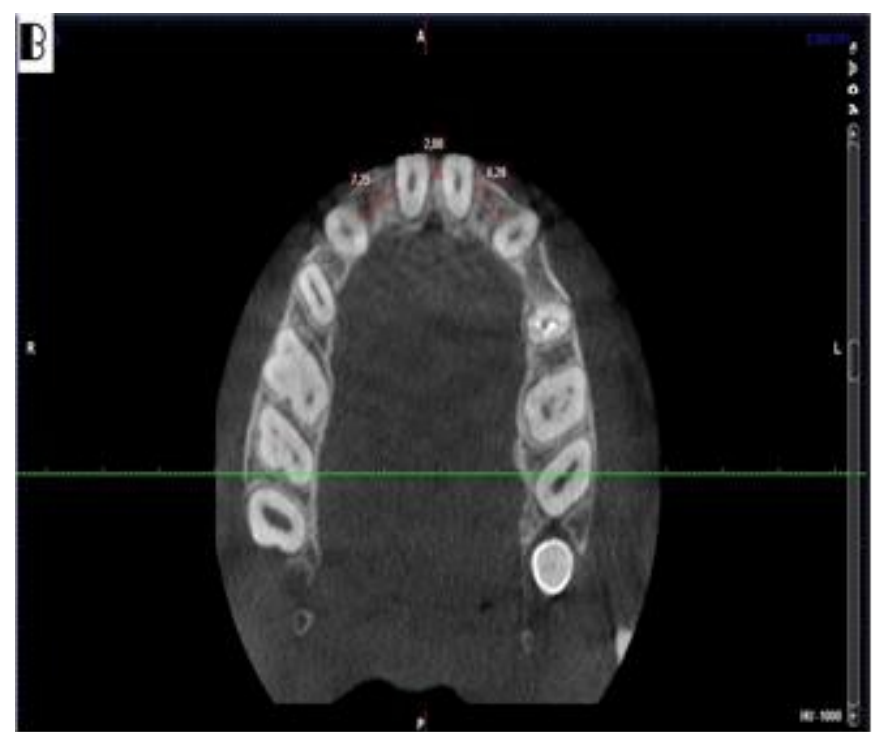

Fig. 4B) The representation of the available space at coronal level between incisors and canines, on both quadrants: $1.3->1.1(7.25 \mathrm{~mm}) ; 1.1->2.1(2 \mathrm{~mm}) ; 2.1->2.3(6.28 \mathrm{~mm})$ indicates, despite the presence of diastema, that the existent space is adequate at this level.

In order to establish precisely whether an implant could be placed at this site, a representation of the apical level is needed.

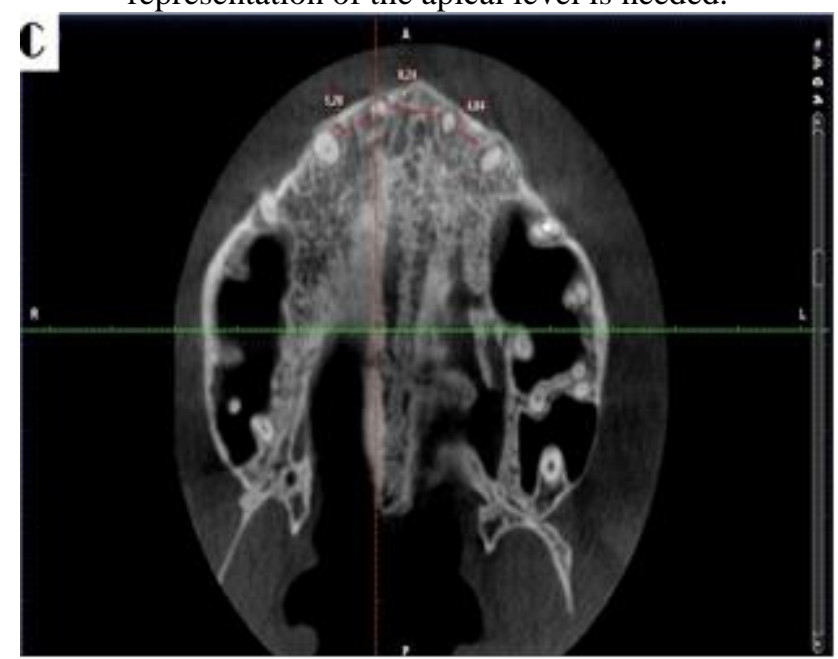

Fig. 5C) The representation of the available space in the apical region points out the inssuficiency of space at this level. A decrease of space is registered apically between the central incisor and the canine $(5.28 \mathrm{~mm}$ and $4.84 \mathrm{~mm})$ and an increase of interincisal space $(8.24 \mathrm{~mm})$, which proves the distal angulation of the roots of the central incisors.

Following the determinations made on each of the 17 examined ortopantomographs, 23 upper central incisors (from the 34 examined teeth) presented a degree of distal root angulation $(67,64 \%)$. The root angulation amplitude was between $3^{\circ}$ (the minimum of angulation taken into account) and $19^{\circ}$ (the maximum encountered angulation). 
The limitations of the present study are associated with the relative small number of examined ortopantomographs and also the use of panoramic radiographs is limited since it describes two-dimensional an threedimensional anatomic structure. Bouwens et. al. (2011) demonstrated that the measurements made on panoramic radiographs are different from those realized with CBCT when examine the same anatomic structures.

Future investigations in an multicentric, epidemiological study, using 3D computed tomography should be conducted to further clarify the results of this study.

\section{Conclusion}

The measurements made on ortopantomographs revealed in most cases of bilateral maxillary lateral incisor agenesis, the modification of the central incisor morphology due to crown-root angulation.

In conclusion, agenesis cases should be very careful investigated, clinical and radiological, using 3D radiological technology. All these cases need a very good interdisciplinary collaboration between the general dentist, the orthodontist, the specialist in radiology, the implantologist and the specialist in prosthodontics respecting the TEAM principle ("together everyone achieves more"). A significant distal corono-radicular angulation will result in an insufficient apical space for the implant and, consequently, an increased risk of radicular fracture during implant insertion. Thereby, a correct radiological evaluation of the position and morphology of the maxillary central incisors is essential for the success of the interdisciplinary treatment.

\section{References:}

1. Abu-Hussein M. et al. (2014). Treatment of Patients With Congenitally Missing Lateral Incisors: Is an Interdisciplinary Task. Research and Reviews: Journal of Dental Sciences; 2: 53-68.

2. Azzaldeen A. et al. (2016). Unilateral Maxillary Lateral Incisor Agenesis with Mini Implant Prostheses: A Case Report. Dental Implants Dentures; 1:106.

3. Bassiouny D.S. et al. (2016) Prevalence of maxillary lateral incisor agenesis and associated skeletal characteristics in an orthodontic patient population. Acta Odontologica Scandinavica; 74(6):456-9.

4. Bouwens D.G., Cevidanes L., Ludlow J.B., Philips C. (2011), Comparison of mesiodistal root angulation with posttreatment panoramic radiographs and cone-beam computed tomography. Am J Orthod Dentofacial Orthop. 139(1): 126-32

5. Celikoglu M., et al. (2012). Investigation of the maxillary lateral incisor agenesis and associated dental anomalies in an orthodontic 
patient population. Medicina Oral Patologoa Oral y Cirugia Bucal; 17(6): e1068-e1073.

6. Kanavakis G. et al. (2015). Evaluation of crown-root angulation of lateral incisors adjacent to palatally impacted canines. Prog Orthod 16(4) DOI 10.1186/s40510-015-0074-0

7. Kinzer G.A., Kokich V.O. (2005) Managing congenitally missing lateral incisors. Part I: canine substitution. Journal of Esthetic and Restorative Dentistry; 17:5-10.

8. Kokich V.G. (2004). Maxillary lateral incisor implants: planning with the aid of orthodontics. Journal of Oral and Maxillofacial Surgery; 62: 48-56.

9. Kokich V.O. Jr., Kinzer G.A. (2005). Managing congenitally missing lateral incisors PartII: Tooth-supported restorations. Journal of Esthetic and Restorative Dentistry; 17:76-84.

10. Larmour C.J. et al. (2005). Hypodontia-A retrospective review of prevalence and etiology. Part I. Quintessence International; 36(4):263-270

11. Pemberton T.J., Das P., Patel P.I. (2005). Hypodontia: genetics and future perspectives. Brazilian Journal of Oral Sciences; 4(13):695706.

12. Pinho T. (2011). Maxilarry lateral incisor agenesis (MLIA). Principles in Contemporary Orthodontics - edited by Dr. Silvano Naretto, ISBN: 978-953-307-687; (2):277-308

13. Stamatiou J., Symons A.L. (1991). Agenesis of the permanent lateral incisor: Distribution, number and sites. The Journal of Clinical Pediatric Dentistry 15(4):244-246

14. Ștefănescu R., Ogodescu A. (2013). Morfologia corono-radiculară a incisivului central superior la pacienții cu anodonție de incisiv lateral maxilar. Lucrare de licență, U.M.F. "Victor Babeș", Timișoara 